九州大学学術情報リポジトリ

Kyushu University Institutional Repository

Particle simulation for predicting fiber motion in injection molding of short-fiber-reinforced composites

Yashiro, Shigeki

Department of Mechanical Engineering, Shizuoka University

Sasaki, Hideaki

Composite Materials Research Laboratories, Toray Industry, Inc.

Sakaida, Yoshihisa

Department of Mechanical Engineering, Shizuoka University

http://hdl. handle. net/2324/4476059

出版情報 : Composites Part A: Applied Science and Manufacturing. 43 (10)，pp.1754-1764，201205-14. Elsevier

バージョン:

権利関係 : 


\section{Particle simulation for predicting fiber motion in injection molding of short-fiber-}

\section{reinforced composites}

Shigeki Yashiro ${ }^{a, *}$, Hideaki Sasaki ${ }^{b}$, and Yoshihisa Sakaida ${ }^{a}$

${ }^{a}$ Department of Mechanical Engineering, Shizuoka University

3-5-1 Johoku, Naka-ku, Hamamatsu 432-8561, Japan

${ }^{\mathrm{b}}$ Composite Materials Research Laboratories, Toray Industry, Inc.

1515, Tsutsui, Masaki-cho, Iyogun, Ehime 791-3193, Japan

* Corresponding author: Tel: +81-53-478-1026; Fax: +81-53-478-1026.

E-mail address: tsyashi@ipc.shizuoka.ac.jp (S. Yashiro)

\section{Abstract}

This study proposes a numerical analysis for predicting fiber motion during injection molding of short-fiber-reinforced composites using the moving particle semi-implicit (MPS) method. Its meshless and Lagrangian nature enables us to track individual fibers and to easily represent free surfaces. In this study, the mechanism of fiber orientation in a T-shaped bifurcation was investigated experimentally and numerically. The fiber orientation of injection-molded glass-fiber/polypropylene composite was observed by X-ray CT. Despite the symmetric mold shape, there was asymmetric fiber orientation due to the mold filling process. Fiber motion in the bifurcation was then analyzed by the proposed simulation, and the fiber orientation was quantitatively evaluated in each small region. The prediction agreed well with the experiment, and the associated mechanism of fiber orientation is discussed. Furthermore, this approach explicitly demonstrates the interaction between fibers, which is an advantage of the proposed approach. 
Keywords: A. Polymer-matrix composites (PMCs); B. Microstructures; C. Numerical analysis; E. Injection moulding.

\section{Introduction}

Fiber-reinforced plastics have recently been applied to structures to reduce their weight. Injection-molded short-fiber-reinforced composites have the advantages of excellent molding flexibility and a short mold cycle time, in addition to a certain level of stiffness and strength. The mechanical properties of injection-molded short-fiber-reinforced composites significantly depend on the condition of the fibers, i.e., their length, dispersion, and orientation. The microscopic structure is governed by the molding process. Accordingly, numerical analyses to predict fiber orientation have been conducted.

Advani and Tucker [1] proposed orientation tensors that could represent fiber orientations with low calculation cost, and developed equations to predict changes in the orientation tensors due to the flow field. Fiber orientations in a thin plate, a weld-line, and a rib structure have been analyzed by using orientation tensors, and these predictions agreed well with observations of injection-molded composites [2-5]. Theoretical and numerical studies have been conducted to consider changes in the flow field due to fiber suspension [6-8]. Improvement of the closure approximation to obtain a higher-order orientation tensor, which is needed to analyze a change in the fiber orientation, has been studied $[9,10]$, and several types of approximations have been evaluated experimentally and numerically $[11,12]$. Thus, orientation tensors have widely been used to analyze fiber orientation. However, numerical methods based on orientation tensors [1-12] cannot analyze more detailed microscopic structures, such as a heterogeneous distribution of fibers.

Fiber condensation and fiber breaks sometimes occur during the injection-molding process, 
and the expected mechanical properties cannot be obtained. In particular, these phenomena can frequently be observed when injecting fibers a few millimeters long into a thin cavity. Therefore, an approach that predicts the motion of individual fibers and the interaction between fibers is needed for accurately predicting material properties. Yamamoto et al. $[13,14]$ proposed a particle simulation to analyze the motion of all fibers, in which each fiber was modeled as an assembly of particles. Although this approach can represent a heterogeneous distribution of fibers, the flow field and the fiber motion were analyzed separately, and thus the effect of fiber motion on the flow field could not be considered.

In recent years, some molding simulations using smoothed particle hydrodynamics (SPH), which is a meshless and fully Lagrangian particle-simulation method, have been demonstrated for die casting [15], polymer processing [16], and concrete [17]. Moreover, Comas-Cardona et al. [18] proposed an analysis that combined SPH with the finite-element method and predicted resin flow during resin-transfer molding of a sandwich structure consisting of glass fabric and foam core. However, to the authors' knowledge, no particle-based simulation of the fiber motion and resin flow of short-fiber-reinforced composites has been presented.

This study, then, proposes a mold-filling simulation of short-fiber-reinforced composites using the moving particle semi-implicit (MPS) method [19]. The MPS method enables us to track the motion of individual fibers and to easily represent free surfaces such as flow-fronts because of its meshless and fully Lagrangian nature. Moreover, if all fibers and resin are modeled by particles, the interactions between fibers, as well as between the fibers and the resin, can be considered automatically.

In this study, fiber orientation in a T-shaped bifurcation area of glass-fiber/polypropylene injection-molded composite was predicted using the MPS method. This paper is organized as follows. Section 2 evaluates the fiber orientation in a T-shaped bifurcation area of an injection 
molded composite by X-ray CT. Section 3 provides a summary of the MPS method and a representation of the reinforcing fibers. Finally, Section 4 presents a particle model of the bifurcation area and analyzes the fiber motion during injection molding. The predicted fiber orientation is quantitatively compared with the experiment, and the mechanism of fiber orientation is discussed.

\section{Experiment}

The material used was an injection-molded composite with glass fibers (GF) and polypropylene (PP). The volume fraction of glass fibers was $8.3 \%$, and the density of the composite was $1030 \mathrm{~kg} / \mathrm{m}^{3}$. A schematic diagram of the flow path near the observed region is presented in Fig. 1. The right $(+y)$ path was terminated $50 \mathrm{~mm}$ away from the branch point, and the left $(-y)$ path was connected with the following path. The average width of all paths was $5 \mathrm{~mm}$. The mold temperature was set to $140^{\circ} \mathrm{C}$ to prevent solidification of the resin and to observe the fiber orientation due only to the resin flow.

The orientation of the glass fiber was investigated by X-ray CT and image processing. Figure 2a presents an X-ray CT image of the bifurcation area; glass fibers are represented by white lines in this image. Next, the fibers were thinned and branched lines were removed by image processing to extract the glass fibers. The observed region was then divided into twelve small areas, in which the probability of fibers was constant, to remove the influence of the image resolution, and the lengths and angles of all fibers were measured in each small area. The orientation angle was defined as the angle between the inflow $(x)$ direction and the longitudinal direction of a fiber. Finally, the fiber orientation distribution in each area, specified as the fraction of fibers within a given angular range, was calculated for every $10^{\circ}$ range. Here, the orientation distribution was obtained by dividing the sum of the fiber lengths 
within a given angular range by the sum of all fiber lengths in the area.

Figure 3 depicts the orientation distribution observed in the inlet region. Fibers were mainly oriented at $0^{\circ}\left(=180^{\circ}\right)$ along the mold wall in the side areas A and $\mathrm{C}$ (Figs. 3a and 3c). However, fibers were randomly oriented in center area B (Fig. 3b). Figure 4 compares the orientation distributions of the center areas of bifurcation, E, H, and K. Fibers were randomly oriented in area E (Fig. 4a), similarly to the neighboring area B. A peak appeared near the $90^{\circ}$ direction in area $\mathrm{H}$ (Fig. 4b), and the peak became sharp and high in the bottom area K (Fig. 4c). The fraction of fibers in the $90^{\circ}$ direction increased with decreasing distance to the bottom wall.

Figure 5 plots the orientation distributions in the corner areas. In the left corner D (Fig. 5a), fibers existed within an angular range of $70^{\circ}$ to $180^{\circ}$, which represents the resin flow along the corner. However, in the right corner (Fig. 5b), fibers were mainly oriented over a range of $0^{\circ}$ to $40^{\circ}$, and the fiber orientations were completely different between the two corners, despite the symmetric mold shape. Similar asymmetric orientation distributions were observed in other areas. The angle at which fibers mostly aligned was different between areas $\mathrm{G}$ and I, which were the center areas of the inlets to the $\pm y$ paths. Although the highest fraction was observed at $90^{\circ}$ in the left area G (Fig. 5c), fibers existed in a wider angular range around $120^{\circ}$ in the right area I (Fig. 5d). Fibers were mostly aligned to the $90^{\circ}$ direction in areas $\mathrm{J}$ and L (Figs. 5e and 5f), which were in contact with the bottom surface; in addition, fibers in the range of $120^{\circ}$ to $130^{\circ}$ were observed in area $\mathrm{L}$.

\section{Moving particle semi-implicit (MPS) method}

\subsection{Numerical approach for incompressible flow}

A continuum is represented by an assembly of particles in the MPS method. A particle 
nearer to particle $i$ is considered to have a greater influence on particle $i$, and the following weight function $w$ is defined to represent the magnitude of the interaction at a distance $r$ from particle $i$.

$$
w(r)=\left\{\begin{array}{cl}
r_{e} / r-1 & \left(0<r \leq r_{e}\right) \\
0 & \left(r_{e}<r\right)
\end{array}\right.
$$

The effects of particles within radius $r_{e}$ from particle $i$ are then considered when analyzing the motion of particle $i$. The sum of weight functions, called the particle number density (PND) $n$, is given by

$$
n_{i}=\sum_{j \neq i} w\left(\left|\mathbf{r}_{j}-\mathbf{r}_{i}\right|\right)
$$

where $\mathbf{r}$ is the position vector and the subscript indicates the number of a particle. PND is proportional to the density of the fluid. Since the density of the fluid is constant for incompressible flow, PND should take a constant value $n_{0}$.

The MPS method analyzes the governing differential equations by using particle interaction models that correspond to differential operators. When a scalar variable $\phi_{i}$ is stored in particle $i$, the gradient of $\phi$ at the position of particle $i$ is represented as

$$
\langle\nabla \phi\rangle_{i}=\frac{d}{n_{0}} \sum_{j \neq i}\left[\frac{\phi_{j}-\phi_{i}}{\left|\mathbf{r}_{j}-\mathbf{r}_{i}\right|^{2}}\left(\mathbf{r}_{j}-\mathbf{r}_{i}\right) w\left(\left|\mathbf{r}_{j}-\mathbf{r}_{i}\right|\right)\right],
$$

where the bracket \langle\rangle indicates the operator based on the particle interaction model and $d$ is the dimension number. Equation (3) is the weighted average of the gradient vector between particles $i$ and $j$ considering the dimension number. The Laplacian model is defined as follows.

$$
\begin{aligned}
& \left\langle\nabla^{2} \phi\right\rangle_{i}=\frac{2 d}{\lambda n_{0}} \sum_{j \neq i}\left[\left(\phi_{j}-\phi_{i}\right) w\left(\left|\mathbf{r}_{j}-\mathbf{r}_{i}\right|\right)\right] \\
& \lambda=\sum_{j \neq i}\left|\mathbf{r}_{j}-\mathbf{r}_{i}\right|^{2} w\left(\left|\mathbf{r}_{j}-\mathbf{r}_{i}\right|\right) / \sum_{j \neq i} w\left(\left|\mathbf{r}_{j}-\mathbf{r}_{i}\right|\right)
\end{aligned}
$$

In the Laplacian model, a part of variable $\phi_{i}$ is divided among neighboring particles considering the weight function, and $\lambda$ is a constant to make the increase in statistical 
dispersion conform to the analytical solution.

The governing equations for incompressible flow are the conservation of mass and the Navier-Stokes equation.

$$
\begin{aligned}
& \frac{D \rho}{D t}=0 \\
& \frac{D \mathbf{u}}{D t}=-\frac{1}{\rho} \nabla P+v \nabla^{2} \mathbf{u}
\end{aligned}
$$

$\rho$ is the density, $\mathbf{u}$ is the velocity, $P$ is the pressure, $v$ is the dynamic coefficient of viscosity, and $\mathrm{D} / \mathrm{D} t$ denotes the Lagrangian differential. In order to apply a semi-implicit solving algorithm, the MPS method uses the mass-conservation equation for compressible flow instead of Eq. (6).

$$
\frac{D \rho}{D t}+\rho \nabla \cdot \mathbf{u}=0
$$

When the position $\mathbf{r}$, velocity $\mathbf{u}$, and pressure $P$ of all particles are known at time $t$, the following semi-implicit algorithm is applied to calculate physical quantities at the new time step $t+\Delta t$. First, temporary values for the velocity and position are explicitly calculated from only the second term of Eq. (7). The temporary PND distribution is not uniform, although the PND must be constant, as shown by Eq. (6). Adjustments in the velocity to keep the density constant are assumed to be caused by the first term of Eq. (7), and Poisson's equation on pressure is then obtained from Eq. (8). The pressure is solved implicitly, and the adjustment in the velocity is calculated from the pressure distribution. Finally, the velocity and the position are updated to make the distribution of the PND uniform. The above semi-implicit algorithm has been described in previous studies $[19,20]$.

Free surfaces can easily be detected using the PND. The PND of a particle on a free surface is smaller than that in a body, since no particle exists outside of the free surface. Therefore, a particle is considered to be on a free surface if its PND is smaller than a constant value 
$\left(0.97 n_{0}\right.$ in this study) after the explicit calculation. The pressure of these particles is zero, and this condition is imposed when solving the Poisson's equation of pressure.

\subsection{Modeling of fibers}

Fibers are modeled as rigid bodies explicitly interacting with an incompressible fluid [19]. Rigid bodies are represented by connecting particles. The position of the center of gravity, $\mathbf{r}_{g}$, and the moment of inertia, $I$, of a rigid body consisting of $N$ particles are given by

$$
\begin{aligned}
& \mathbf{r}_{g}=\frac{1}{N} \sum_{i=1}^{N} \mathbf{r}_{i}, \\
& I=\sum_{i=1}^{N} m\left|\mathbf{r}_{i}-\mathbf{r}_{g}\right|^{2},
\end{aligned}
$$

where $m$ is the mass of a particle. Weak interaction between the fluid and the rigid bodies is assumed, and all particles are first moved, based on Eqs. (6) and (7), without distinguishing rigid bodies from the fluid at time step $(k+1)$. The change in the center of gravity of a rigid body from the time step $k$ to $(k+1), \mathbf{r}_{g}^{\prime}$, is calculated as

$$
\mathbf{r}_{g}^{\prime}=\frac{1}{N} \sum_{i=1}^{N}\left(\hat{\mathbf{r}}_{i}^{k+1}-\mathbf{r}_{i}^{k}\right),
$$

where $\hat{\mathbf{r}}_{i}^{k+1}$ denotes the position of particle $i$ after the implicit calculation for fluid at step $(k+1)$. The change in rotation angle $\theta^{\prime}$ is obtained from the relation between the angular momentum and the angular velocity.

$$
\theta^{\prime}=\frac{1}{I} \sum_{i=1}^{N} m\left(\mathbf{r}_{i}^{k}-\mathbf{r}_{g}^{k}\right) \times\left(\hat{\mathbf{r}}_{i}^{k+1}-\mathbf{r}_{i}^{k}\right)
$$

The relative position between the particles in the rigid body is then corrected to the original shape while retaining the changes in the position of the center of gravity and the rotation angle. Here, the position change of particle $i, \mathbf{r}^{\prime}{ }_{i}$, is given by 


$$
\mathbf{r}_{i}^{\prime}=-\left(\mathbf{r}_{i}^{k}-\mathbf{r}_{g}^{k}\right)+\mathbf{r}_{g}^{\prime}+\left[\begin{array}{cc}
\cos \theta^{\prime} & -\sin \theta^{\prime} \\
\sin \theta^{\prime} & \cos \theta^{\prime}
\end{array}\right]\left(\mathbf{r}_{i}^{k}-\mathbf{r}_{g}^{k}\right)
$$

Accordingly, the velocity and the position of particle $i$ at time step $(k+1)$ are calculated as follows.

$$
\begin{aligned}
& \mathbf{u}_{i}^{k+1}=\frac{\mathbf{r}_{i}^{k+1}-\mathbf{r}_{i}^{k}}{\Delta t}=\frac{\mathbf{r}_{i}^{\prime}}{\Delta t} \\
& \mathbf{r}_{i}^{k+1}=\mathbf{r}_{i}^{k}+\mathbf{r}_{i}^{\prime}
\end{aligned}
$$

The above calculations for rigid bodies correspond to analyzing the motion of a rigid body by integrating the force on particles in the rigid body affected by the fluid and other rigid bodies.

The MPS method considers the equilibrium of force induced by interactions between resin and resin (i.e., a flow field), between a fiber and resin, and between a fiber and a fiber by solving Eqs. (6) and (7). Two particles repel each other when they move closer, and they cannot overlap. Similarly, two fibers repel each other when they move closer, and the particles in the fibers move due to the generated force and moment. Thus, the fiber-fiber interaction is considered automatically when solving the governing equations and is observed in the particle motion. It should be noted that the other interaction effects require additional interaction models in Eq. (7).

\section{Analysis}

\subsection{Verification of the numerical approach}

The present approach was verified by comparing the prediction with a theoretical solution. Incompressible fluid with viscosity of $200 \mathrm{~Pa} \cdot \mathrm{s}$ was injected in a straight path with a width of $5 \mathrm{~mm}$. In order to remove the influence of the initial condition on the flow front, the flow path was bent toward the $x$-direction following injection in the $y$-direction; the injection speed was $0.5 \mathrm{~m} / \mathrm{s}$. The initial distance between particles was $0.25 \mathrm{~mm}$. Figure 6 depicts the pressure 
distribution. The pressure gradient was almost constant $\left(\mathrm{d} P / \mathrm{d} x=-3.9 \times 10^{7}\right)$ between the position without velocity in the $y$-direction and the flow front $(6.5<x<32 \mathrm{~mm})$ despite wide variations in the pressure. The predicted velocity in the $x$-direction is compared with the theory for Poiseuille flow in Fig. 7. The prediction agreed with the theory; the error in the velocity at the center was $8.7 \%$, caused by the pressure fluctuation and the accuracy of the differential operator models for the MPS method. Figure 8 depicts the distribution of the velocity relative to that of a particle at the flow front. The velocity distribution exhibited a fountain flow at the flow front. These results demonstrate the validity of the MPS method.

The motion of a single fiber was analyzed in a simple shear flow with a shear rate $\dot{\gamma}$ of $500 \mathrm{~s}^{-1}$, and the prediction accuracy of the orientation angle was investigated. Figure 9a depicts the analytical model for the simple shear flow with a single rigid fiber; the initial distance between particles was $0.25 \mathrm{~mm}$. A rigid fiber (connected particles) was allocated at the center of the path at the initial orientation angle of $90^{\circ}$ from the $x$-direction. The path width $2 h$ exceeded the fiber length by three times, and the path length was four times greater than the path width. The upper and lower plates had a velocity of $\pm \dot{\gamma} h$, and the velocity of the simple shear flow $\left(v_{x}, v_{y}\right)=( \pm \dot{\gamma} y, 0)$ is assigned to all of the particles as the initial velocity. The periodic boundary condition was imposed on both ends of the path. The fiber density was $2540 \mathrm{~kg} / \mathrm{m}^{3}$, and the viscosity and density of the fluid were $200 \mathrm{~Pa} \cdot \mathrm{s}$ and 900 $\mathrm{kg} / \mathrm{m}^{3}$. Figure $9 \mathrm{~b}$ plots the velocity in the $x$-direction at the center of the path (fiber aspect ratio of 10). Although a sparse particle distribution was generated due to the fluctuation of pressure [20], the velocity distribution agreed with the solution for simple shear flow.

Figure 10 plots the predicted orientation angle $\phi$ for aspect ratio $r_{e}$ of 4, 10, 20, and 40; the dashed line represents the fiber orientation analyzed by the Jeffery's model [21]. The Jeffery's solution for simple shear flow is simplified as follows [22], where $\phi_{0}$ is the initial 
orientation angle.

$$
\tan \phi=\frac{1}{r_{e}} \tan \left[-\dot{\gamma} \frac{r_{e}}{r_{e}^{2}+1}+\tan ^{-1}\left(r_{e} \tan \phi_{0}\right)\right]
$$

The prediction agreed with the Jeffery's solution for all fiber-aspect ratios. The errors observed after $0.05 \mathrm{~s}$ were caused by the sparse particle distribution due to the pressure fluctuation, and disappeared in a sufficiently long flow path.

Finally, the orientation state obtained by the present analysis was compared with that predicted by the orientation distribution function [1]. Rigid fibers were injected in the straight path depicted in Fig. 6. The analytical conditions were the same as the above simulations, and the fiber length was $1 \mathrm{~mm}$; the fiber volume fraction $V_{f}$ was $10 \%$ and $40 \%$. The fibers aligned in the $x$-direction were positioned randomly at the inlet. After the path was filled, the angles of the fibers within $10<x<35 \mathrm{~mm}$ were measured. The simulation was performed 10 times, and over 500 fibers were counted to obtain the orientation distribution. The path was divided into the core region with $60 \%$ of the path width and the remaining skin layers, and the orientation distributions in the two regions were evaluated. The orientation states were also predicted using the orientation distribution function. The calculated orientation distributions were then converted to the second-order orientation tensor $a_{i j}[1]$ to compare the orientation states between the present analysis and the conventional method. The component $a_{11}$ equals unity $(0.5)$ at the unidirectional (completely random) orientation, while $a_{i i}=1$. Table 1 lists the orientation tensors obtained by the present analysis. The smaller $V_{f}$ exhibited a stronger trend of the unidirectional orientation. Figure 11 plots the component $a_{11}$ against the interaction coefficient $C_{I}$. The orientation state for $10 \% V_{f}$ suggested $C_{I}<0.002$, which was equivalent to the very week interaction between fibers. The results of $40 \% V_{f}$ corresponded to $C_{I} \approx 0.01 ; C_{I}$ increased with increasing $V_{f}$. These results indicated that the present analysis could represent interaction between fibers. It should be noted that the interaction effect in this 
analysis was smaller than that in the actual molding, because the fiber aspect ratio (number of fibers) was small in this analysis.

\subsection{Analytical model of injection molding}

Figure 12 depicts the analytical model. The $-y$ flow path is longer than the $+y$ flow path to approximate the actual mold as seen in Fig. 1. The wall surface was represented by particles (Fig. 12b), with one inner layer on which the pressure was calculated and two outer layers where the pressure was not calculated. The initial distance between particles, $l_{0}$, was $0.25 \mathrm{~mm}$. The number of layers depends on the effective radius $r_{e}\left(=2.1 l_{0}\right.$ in this study). Three layers were needed so that fluid particles neighboring the wall surface were judged not to be on a free surface.

Particles for polypropylene (PP) and glass fibers (GF) were injected from the upper inflow part at a constant injection speed of $0.5 \mathrm{~m} / \mathrm{s}$ until the mold was filled. The wall of the inflow part moved in the $+x$ direction to push the fluid particles forward, and the moving wall was shifted back to the original position after it had moved the distance $l_{0}$. New particles for polypropylene and glass fibers were introduced in the generated gap. Polypropylene was modeled as an incompressible fluid, with density and viscosity of $900 \mathrm{~kg} / \mathrm{m}^{3}$ and $200 \mathrm{~Pa} \cdot \mathrm{s}$. Glass fibers were modeled as rigid bodies with a density of $2540 \mathrm{~kg} / \mathrm{m}^{3}$. The number of particles for the mold wall was 2256, and that for the moving wall was 60 . There were 10296 particles after the mold was completely filled $(0.20 \mathrm{~s})$. The typical calculation time was 68 hours using a computer with an Intel Xeon CPU at $2.67 \mathrm{GHz}$ with $6 \mathrm{~GB}$ of memory. The memory usage was $613 \mathrm{MB}$.

Among the generated particles at the inflow gate, four neighboring particles were considered to be a glass fiber $1 \mathrm{~mm}$ in length whose position was determined by a random 
number; the other particles represented polypropylene (Fig. 12b). The mold-filling simulation was conducted 25 times because of the use of random numbers.

\subsection{Results and discussion}

Figure 13 presents snapshots of the flow of the resin and fibers. The flow-front reached the bottom of the bifurcation at $0.04 \mathrm{~s}$, and the resin flowed into the $\pm y$ paths. The $+y$ path filled with resin at $0.13 \mathrm{~s}$, and fibers in the $+y$ path then hardly moved after the path was filled. The resin and fibers continued flowing into the $-y$ path till $0.20 \mathrm{~s}$. The resin sprang up from a backward position to the flow-front, and a fountain flow with a convex surface was generated. A sparse distribution of particles, corresponding to an unrealistic free surface in the resin (void), occasionally appeared at the flow front. This may be caused by instability in setting the pressure-free boundary condition. Therefore, it is difficult to discuss detailed phenomena at the flow front using the present approach, although fountain flow was observed in Fig. 8. Unrealistic free surfaces will be removed by considering the surface (interfacial) tension modeled as an external force in Eq. (7). The surface and interfacial tension will be discussed in our next study [23].

Rigid fibers mostly aligned in the flow direction near the side walls. However, random orientations were frequently observed in the middle of the path width. This phenomenon was caused by the gradient of the velocity in the flow direction due to viscosity. Figure 14 depicts the distribution of the shear rate and vorticity at $0.12 \mathrm{~s}$. A high shear rate (vorticity) was generated near the side wall because of the steep velocity gradient, and fibers rotated to become oriented parallel to the side wall. Fibers hardly rotated in the middle region of a path because of the low shear rate (vorticity); instead, random orientation was observed. Thus, the fiber orientation in a straight path was represented qualitatively and agreed with conventional 
knowledge.

In order to quantitatively evaluate the predicted fiber orientation, the bifurcation area was divided into twelve areas as seen in Fig. 2b. The fiber-orientation distribution in each area was calculated after mold filling was finished $(0.20 \mathrm{~s})$, and the orientation angle was measured from the $x$ direction. The predicted results of the fiber angles from 25 simulations were integrated to obtain the orientation distribution. Since the fiber length was constant in this analysis, the orientation distribution could be calculated by dividing the number of fibers in an angular range by the total number of fibers in a given area. The fiber aspect ratio was 4 in the simulation, but the aspect ratio in the experiment was expected to be 50 to 100 . However, the influence of the small aspect ratio on the orientation distribution would be limited, since the Jeffery's solution demonstrates that the change in orientation angle for an aspect ratio of 4 is almost the same as that for a larger aspect ratio when $\phi>20^{\circ}$, and that a given fiber experienced the greatest shear rate $\left(600 \mathrm{~s}^{-1}\right)$ only briefly.

Figure 3 plots the predicted orientation distribution in the inlet region to the bifurcation, along with the experiment results. Most fibers were aligned in the $0^{\circ}$ direction in the side areas (A and C; Figs. 3a and 3c), and fibers were randomly oriented in the middle area (B; Fig. 3b). These predictions agreed well with the observations. Figure 4 plots the orientation distribution in the center areas $(\mathrm{E}, \mathrm{H}$, and $\mathrm{K})$ of the bifurcation. Random orientation was predicted in upstream area $\mathrm{E}$, and the fraction aligned to the $90^{\circ}$ direction increased with decreasing distance to the bottom surface. These predictions also agreed with the experiment results.

Figure 5 depicts the orientation distributions at the corners. As observed in the experiment, the fiber orientation differed between the left and right corner areas despite the symmetric corner shapes. In the left corner (D; Fig. 5a), fibers were oriented over a wide angular range 
of $90^{\circ}$ to $30^{\circ}$ via $180^{\circ}$. However, in the right corner area (F; Fig. 5b), fibers were oriented over a range of $150^{\circ}$ to $20^{\circ}$, which was smaller than that of the left corner, and this tendency agreed with the experiment. This difference was caused by the order of mold-filling, i.e., by the fact that the $+y$ path was completely filled before $-y$ path was filled. Figure 15 presents the velocity vector diagram at $0.16 \mathrm{~s}$ (after the $+y$ path was filled); particles with velocities of $5 \%$ of the injection speed are represented by circles. A plane with minimal velocities (dashed line) appeared near the right corner, and this plane became a temporary wall surface. Fibers were then oriented parallel to the temporary wall $\left(0^{\circ}\right.$ to $\left.20^{\circ}\right)$ in area $\mathrm{F}$, and the angular range over which fibers existed was narrower than that of area D. Thus, the fiber orientation differed between the left and right corners depending on the mold-filling process.

Effects of the temporary wall on the fiber orientation were observed in the other areas in the bifurcation. In the inlet of the $-y$ path, a large fraction was predicted near $90^{\circ}$ in the middle area (G; Fig. 5c) and the bottom area (J; Fig. 5e). However, in the inlet of the $+y$ path, a large fraction was predicted in the range of $100^{\circ}$ to $160^{\circ}$ in the middle area (I; Fig. 5d), with fibers in the range of $120^{\circ}$ to $140^{\circ}$ existing in the bottom area (L; Fig. 5f). These orientation distributions in the right corner were generated by the flow along the temporary wall. Thus, the differences of the fiber orientations between the right and left corners agreed well with the experiment results, and the proposed approach can represent the fiber orientation of injectionmolded composites.

Furthermore, fibers accumulated at some points during and/or after resin flow, as depicted in Fig. $13(0.20 \mathrm{~s})$. Since the flow velocity increased with increasing distance from the side wall due to the viscosity of the resin, fibers with a high velocity collided with low-velocity fibers, which were frequently positioned near a side wall. When these fibers existed in a small area, the fibers could not move freely so they accumulated. This physical interaction between 
fibers disturbed the directions of the fibers, and irregular fiber orientation could appear due to the high fiber content. The explicit representation of the fiber-fiber interaction is an advantage of this particle simulation.

\section{Conclusions}

This study proposed a mold-filling simulation for short-fiber-reinforced composites using the MPS method. All of the fibers were modeled by several continuing particles, so this approach could analyze the motion of a specific fiber and automatically considered the interaction between fibers and resin and between fibers. In this study, fibers (resin) were considered as rigid bodies (an incompressible viscous fluid), and the motion of fibers during the injection-molding process was analyzed. Fiber orientation distributions in a bifurcation were predicted and quantitatively compared with the observed results of fiber orientation in injection-molded GF/PP composites. The conclusions are summarized below.

1. The predicted fiber-orientation distributions agreed well with those observed by X-ray CT in all of the bifurcation areas. These results confirmed the validity of the proposed particle-based simulation.

2. A plane on which the flow velocity almost vanished formed because of the difference in the time required to fill the branched paths. Resin flow along this temporary surface generated a fiber orientation parallel to that surface and resulted in a difference in fiber orientation between the left and right corners despite the symmetric corner shapes. Thus, the mechanism of fiber orientation near a corner was clarified.

3. Direct modeling of fibers by continuing particles enabled us to explicitly reproduce the accumulation of fibers. 


\section{Acknowledgment}

This work is part of the Japanese METI-NEDO project "Development of sustainable hyper composite technology" that commenced in 2008. S. Y. also acknowledges the support of the Ministry of Education, Culture, Sports, Science and Technology of Japan under Grants-in-Aid for Scientific Research (No. 22760524).

\section{Reference}

[1] Advani S G, Tucker C L, The use of tensors to describe and predict fiber orientation in short fiber composites. J Rheol 1987; 31(8):751-784.

[2] Guputa M, Wang K K, Fiber orientation and mechanical properties of short-fiberreinforced injection-molded composites: simulated and experimental results. Polym Compos 1993; 14(5):367-382.

[3] Kim J K, Song J H, Chung S T, Kwon T H. Morphology and mechanical properties of injection molded articles with weld-lines. Polym Eng Sci 1997; 37(1):228-241.

[4] VerWeyst B E, Tucker C L, Foss P H, O'Gara JF. Fiber orientation in 3-D injection molded features: prediction and experiment. Int Polym Proc 1999; 1999(4):409-420.

[5] Kim E G, Park J K, Jo S H. A study on fiber orientation during the injection molding of fiber-reinforced polymeric composites (Comparison between image processing results and numerical simulation). J Mater Process Technol 2001; 111(1-3):225-232.

[6] Chung S T, Kwon T H. Coupled Analysis of injection molding filling and fiber orientation, including in-plane velocity gradient effect. Polym Compos 1996; 17(6):859872.

[7] Chung D H, Kwon T H. Numerical studies of fiber suspensions in an axisymmetric radial diverging flow: the effects of modeling and numerical assumptions. J. Non-Newtonian 
Fluid Mech 2002; 107(1-3):67-96.

[8] Han K-H, Im Y-T. Numerical simulation of three-dimensional fiber orientation in injection molding including fountain flow effect. Polym Compos 2002; 23(2):222-238.

[9] Cintra J S, Tucker C L. Orthotropic closure approximations for flow-induced fiber orientation. J Rheol 1995; 39(6):1095-1122.

[10] Montgomery-Smith S, Jack D, Smith D E. The fast exact closure for Jeffery's equation with diffusion. J Non-Newtonian Fluid Mech 2011; 166(7-8):343-353.

[11] Dray D, Gilormini P, Régnier G. Comparison of several closure approximations for evaluating the thermoelastic properties of an injection molded short-fiber composite. Compos Sci Technol 2007; 67(7-8):1601-1610.

[12] Jack D A, Smith D E. The effect of fibre orientation closure approximations on mechanical property predictions. Compos Part A 2007; 38(3):975-982.

[13] Yamamoto S, Matsuoka T. Dynamic simulation of microstructure and rheology of fiber suspensions. Polym Eng Sci 1996; 36(19):2396-2403.

[14] Yamamoto S, Matsuoka T, Dynamic simulation of rod-like and plate-like particle dispersed systems. Comput Mater Sci 1999; 14(1-4):169-176.

[15]Kulasegaram S, Bonet J, Lewis RW, Profit M. High pressure die casting simulation using a Lagrangian particle method. Commun Numer Meth Engng 2003; 19(9):679-687.

[16]Fan XJ, Tanner RI, Zheng R. Smoothed particle hydrodynamics simulation of nonNewtonian moulding flow. J Non-Newtonian Fluid Mech 2010; 165(5-6):219-226.

[17]Kulasegaram S, Karihaloo BL, Ghanbari A. Modelling the flow of self-compacting concrete. Int J Numer Anal Meth Geomech 2011; 35(6):713-723.

[18] Comas-Cardona S, Groenenboom P, Binetruy C, Krawczak P. A generic mixed FE-SPH method to address hydro-mechanical coupling in liquid composite moulding processes. 
Compos Part A 2005; 36(7):1004-1010.

[19] Koshizuka S, Nobe A, Oka Y. Numerical analysis of breaking waves using the moving particle semi-implicit method. Int J Numer Meth Fluids 1998; 26(7):751-769.

[20] Tanaka M, Masunaga T. Stabilization and smoothing of pressure in MPS method by quasi-compressibility. J Comput Phys 2010; 229(11):4279-4290.

[21] Jeffery G B. The motion of ellipsoidal particles immersed in a viscous fluid. Proc R Soc Lond A 1922; 102(715):161-179.

[22] Moses K B, Advani S G, Reinhardt A. Investigation of fiber motion near solid boundaries in simple shear flow. Rheol Acta 2001; 40(3):296-306.

[23] Okabe T, Matsutani H, Honda T, Yashiro S. Numerical simulation of resin flow using the moving particle semi-implicit method. Composites Part A; submitted for publication.

\section{Figure captions}

Fig. 1 Schematic diagram of the injection mold near the observed area.

Fig. 2 Observation of the bifurcation region for injection-molded GF/PP composite.

Fig. 3 Fiber-orientation distribution at the inlet region of the bifurcation. Fibers were aligned in the $x$-direction near the side walls, and were randomly oriented in the middle of the flow path.

Fig. 4 Fiber-orientation distribution at the center in the $y$-direction. The orientation parallel to the $y$-direction increased with decreasing distance to the bottom wall.

Fig. 5 Fiber-orientation distribution near the corners. The orientation distributions differed between the left and right corners despite the symmetric corner shapes.

Fig. 6 Pressure distribution in a straight flow path at $0.08 \mathrm{~s}$. The pressure decreased linearly. 
Fig. 7 Comparison of the velocity in the $x$-direction with the prediction and the solution of the Poiseuille flow.

Fig. 8 Distribution of the velocity relative to that of a particle at the flow front, which exhibited fountain flow.

Fig. 9 Analytical model for simple shear flow with a single rigid fiber. The path size was changed by the fiber aspect ratio.

Fig. 10 Predicted orientation angle in simple shear flow. The Jeffery's solution is also indicated by dashed lines.

Fig. 11 Change in the orientation tensor component $a_{11}$ against the fiber interaction coefficient calculated at the straight path using the orientation distribution function.

Fig. 12 Analytical model of a mold with a bifurcation. The mold wall consisted of three layers of particles. Fibers were generated at random positions of the inflow particles.

Fig. 13 Snapshots of the mold-filling flow. A fountain flow was generated at the flow-fronts. Fibers accumulated during resin flow.

Fig. 14Distribution of (a) shear rate and (b) vorticity at $0.12 \mathrm{~s}$.

Fig. 15 Velocity vector diagram at $0.16 \mathrm{~s}$ after the $+y$ flow path was filled with resin. Particles with a velocity of $0.025 \mathrm{~m} / \mathrm{s}$ (5\% of the injection speed) are represented by circles. Resin flow almost vanished at the plane indicated by the dashed line.

\section{Table caption}

Table 1 The second-order orientation tensors at the straight path obtained by the present approach. 
Table 1 The second-order orientation tensors at the straight path obtained by the present approach.

\begin{tabular}{cccc}
\hline$V_{f}$ & & $a_{11}$ & $a_{12}$ \\
& Skin & 0.981 & $-1.32 \times 10^{-3}$ \\
$10 \%$ & Core & 0.832 & $-4.66 \times 10^{-2}$ \\
& Skin & 0.957 & $1.66 \times 10^{-2}$ \\
$40 \%$ & Core & 0.791 & $-5.31 \times 10^{-2}$ \\
\hline
\end{tabular}




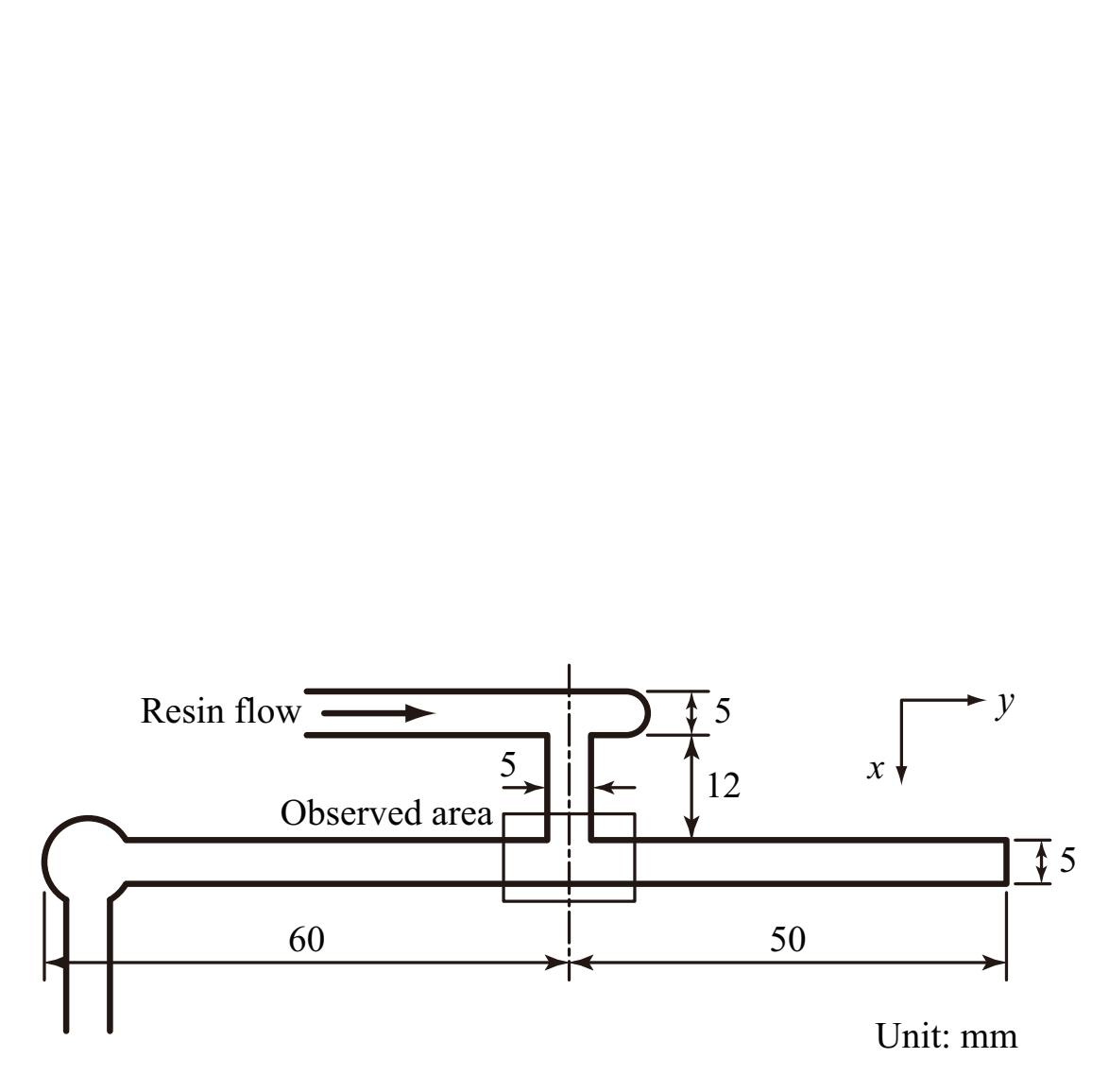

Fig. 1 Schematic diagram of the injection mold near the observed area.

Unit: $\mathrm{mm}$ 


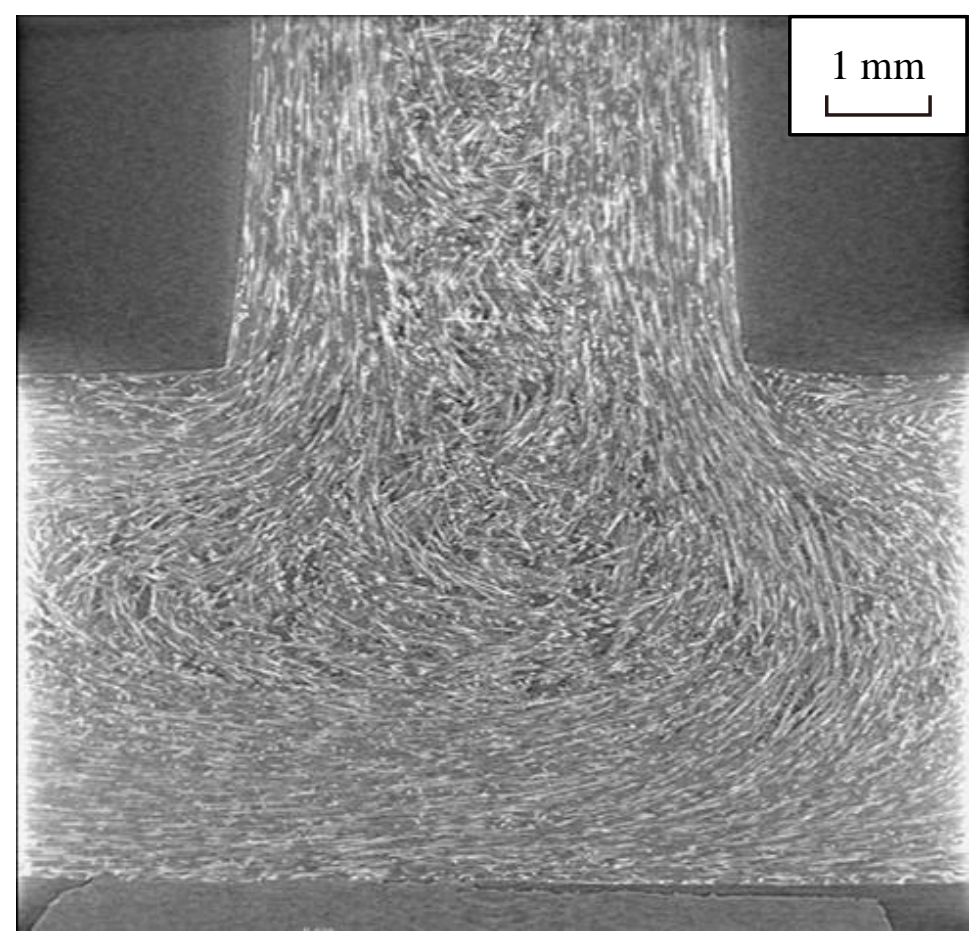

(a) X-ray CT image

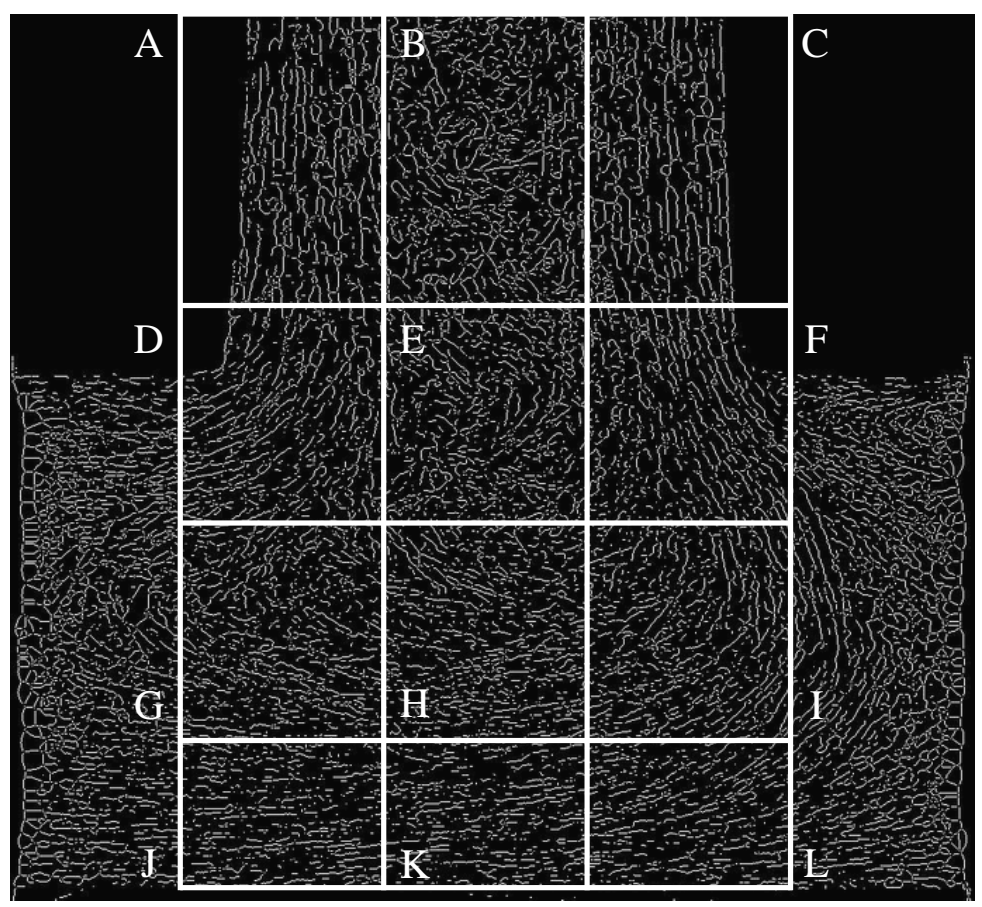

(b) Processed image

Fig. 2 Observation of the bifurcation region for injection-molded GF/PP composite. 


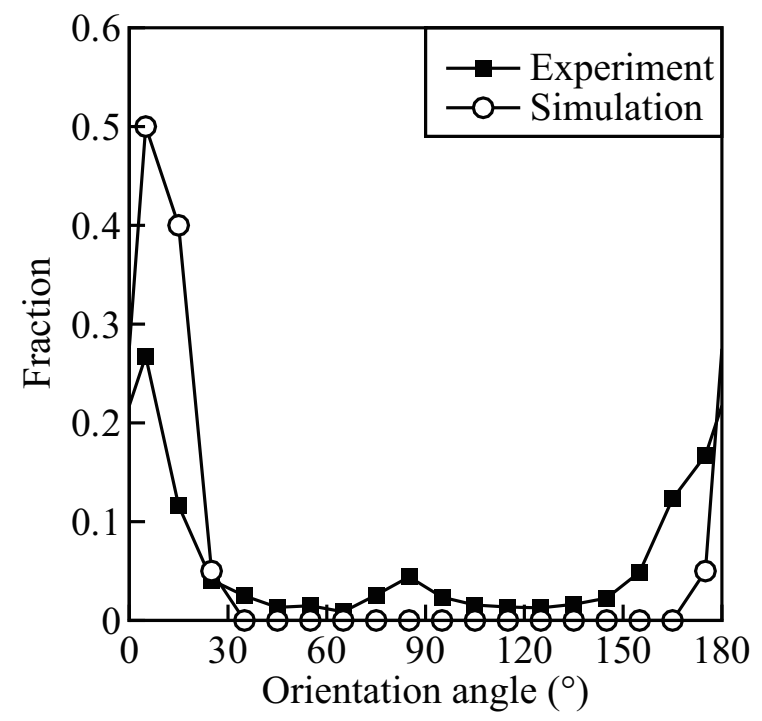

(a) Area A

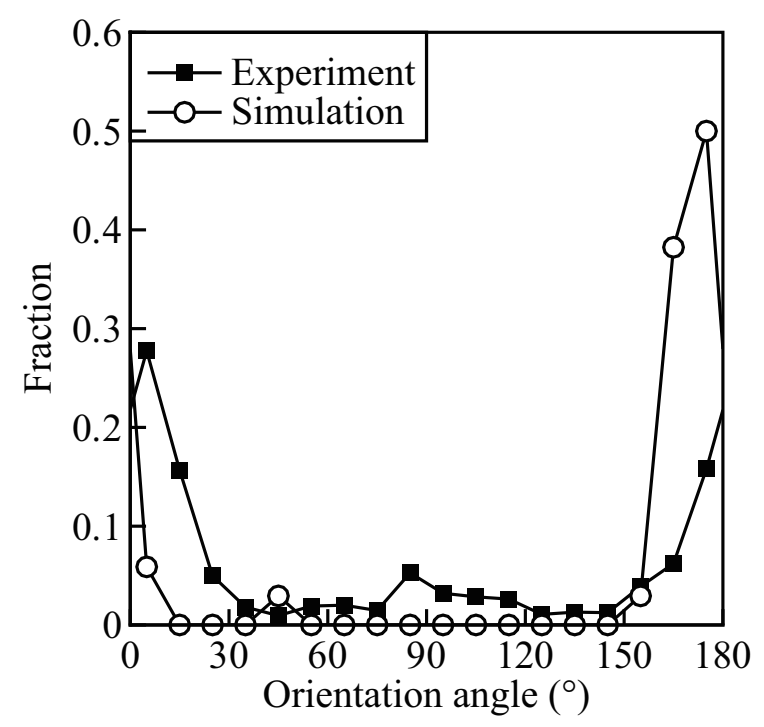

(c) Area C

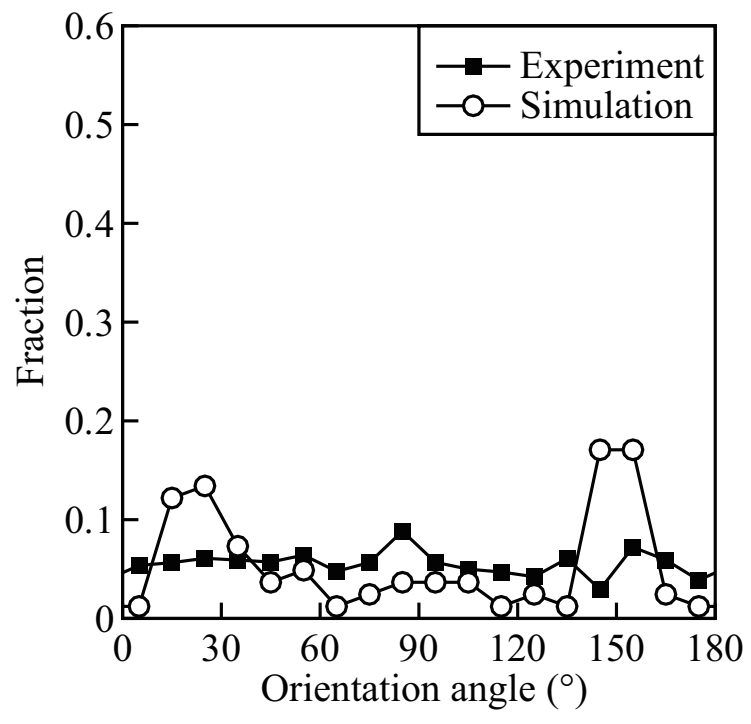

(b) Area B

Fig. 3 Fiber-orientation distribution at the inlet region of the bifurcation. Fibers were aligned in the $x$-direction near the side walls, and were randomly oriented in the middle of the flow path. 


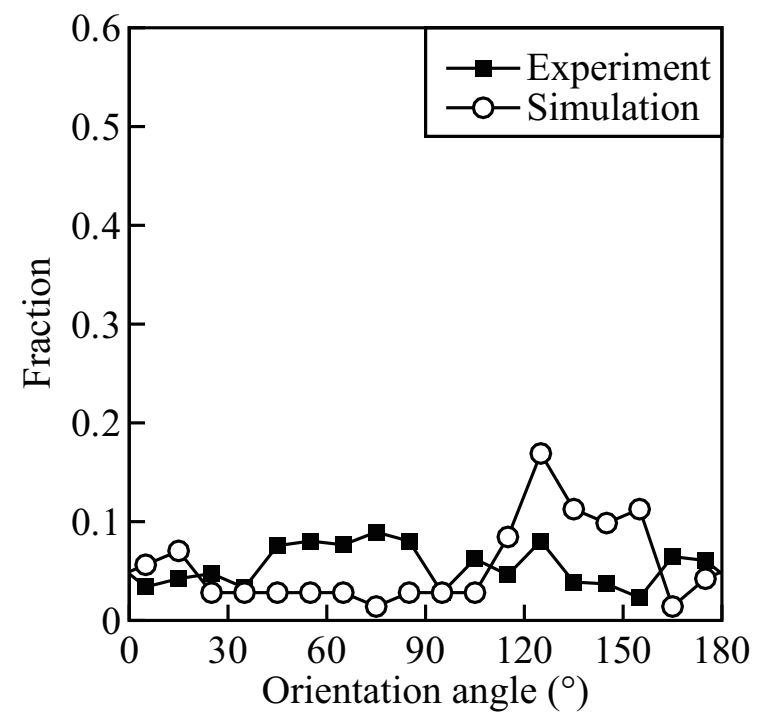

(a) Area E

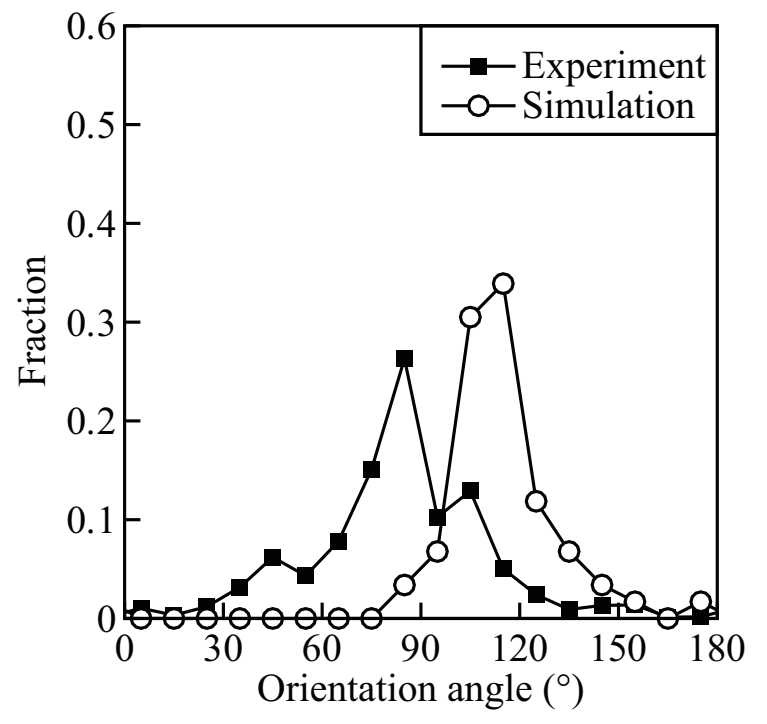

(b) Area $\mathrm{H}$

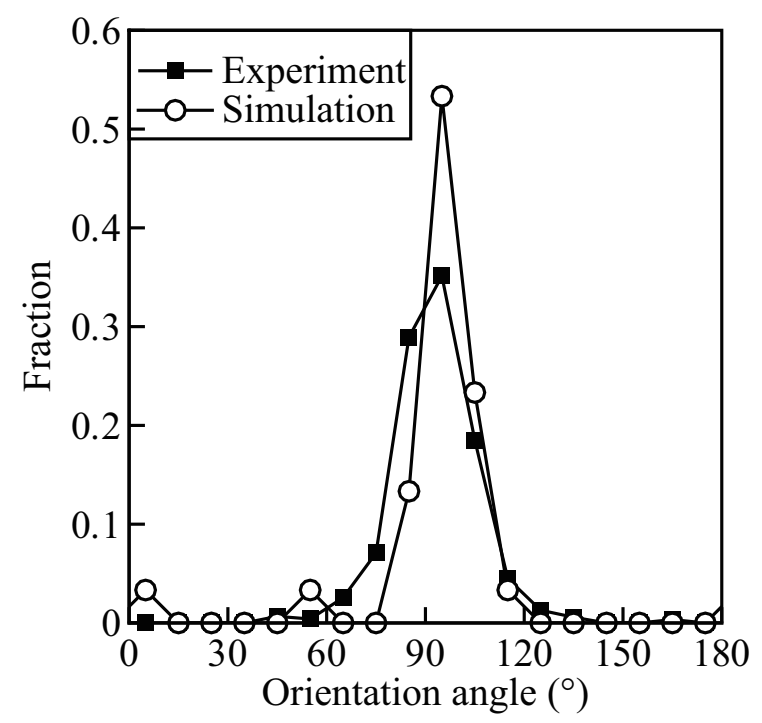

(c) Area K

Fig. 4 Fiber-orientation distribution at the center in the y-direction. The orientation parallel to the $y$-direction increased with decreasing distance to the bottom wall. 


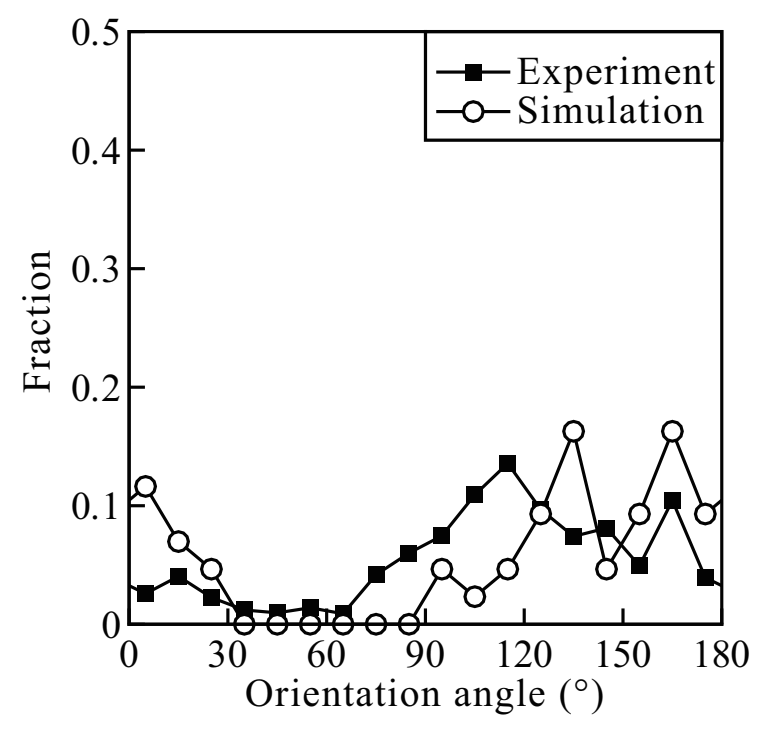

(a) Area D

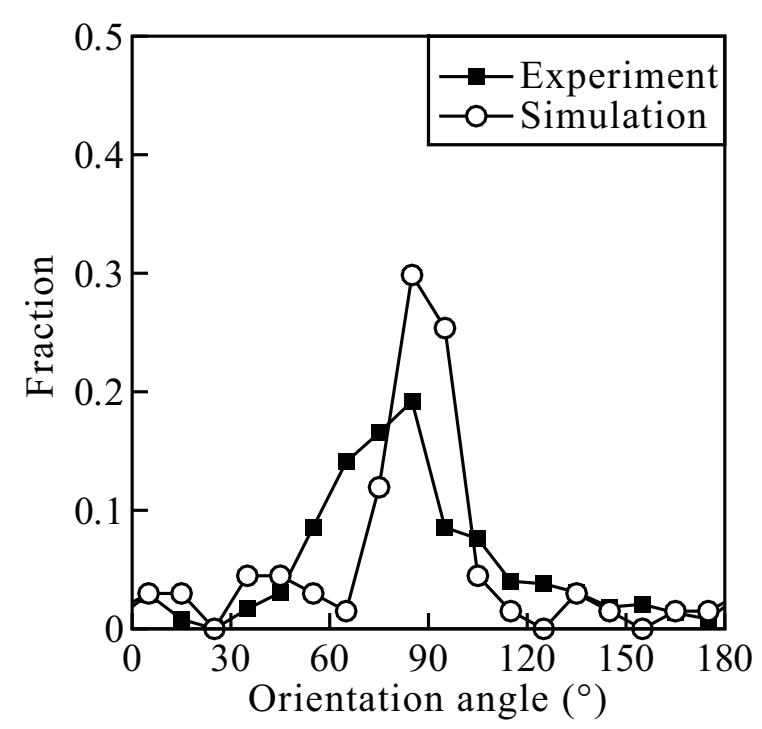

(c) Area G

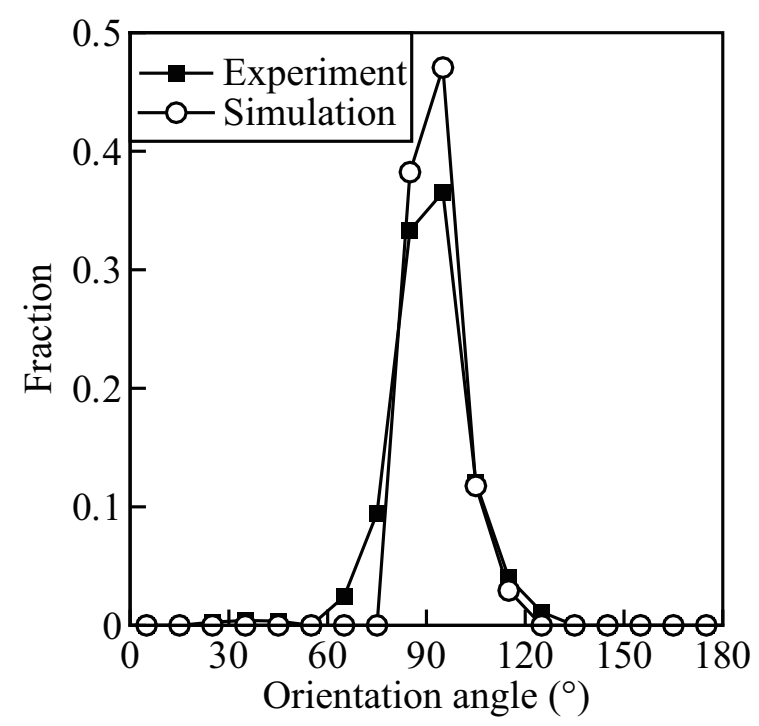

(e) Area J

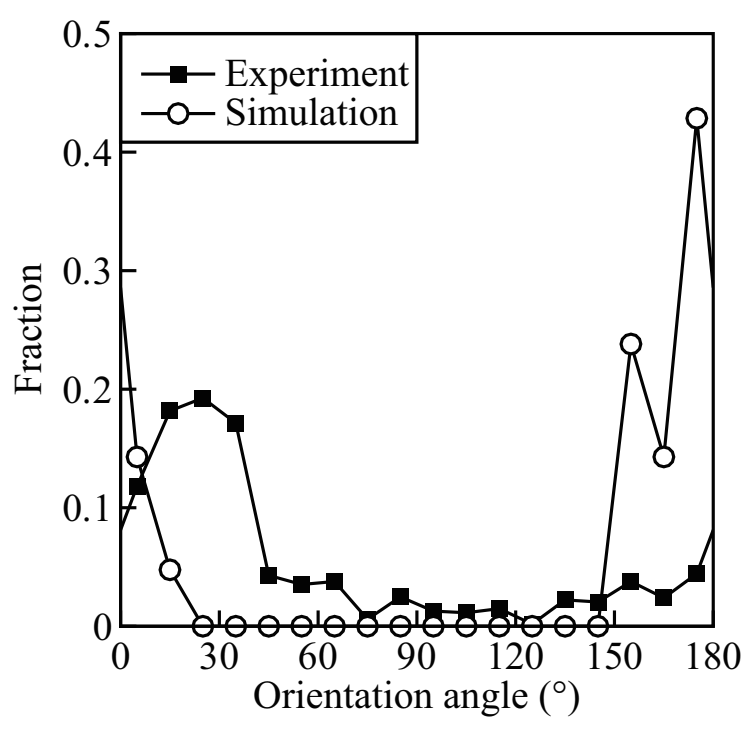

(b) Area F

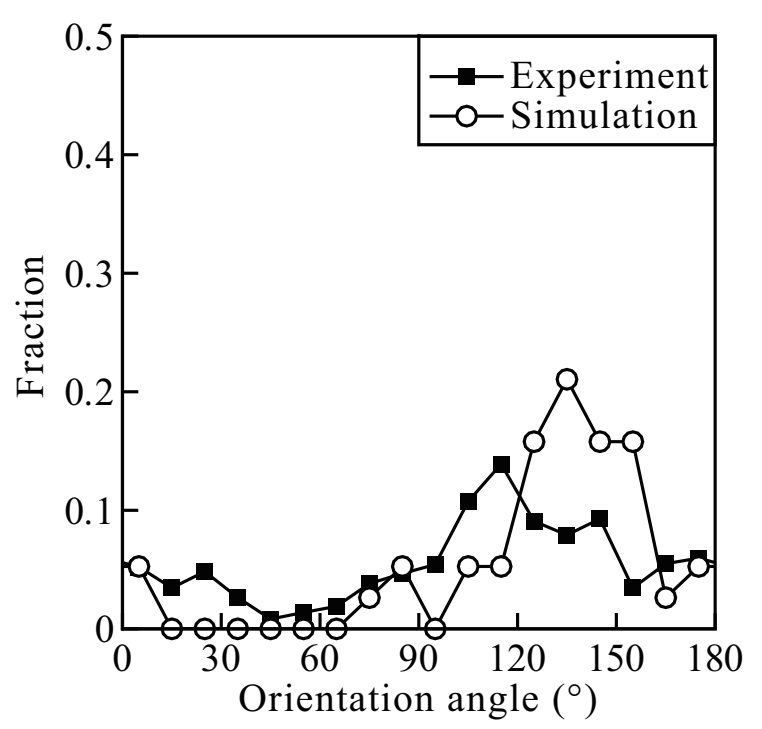

(d) Area I

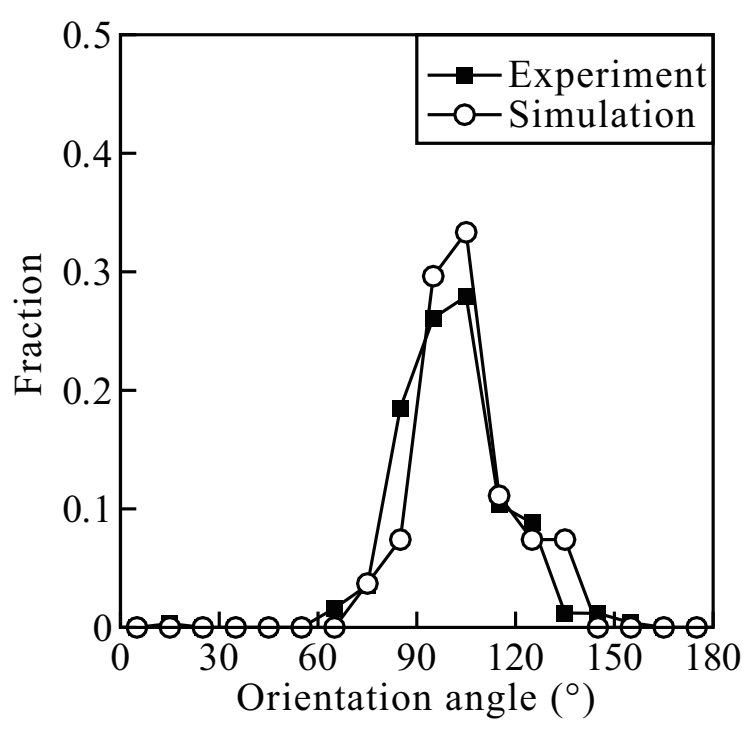

(f) Area L

Fig. 5 Fiber-orientation distribution near the corners. The orientation distributions differed between the left and right corners despite the symmetric corner shapes. 
Pressure $(\mathrm{Pa})$

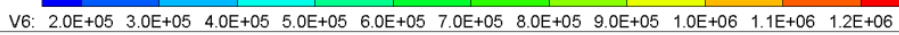

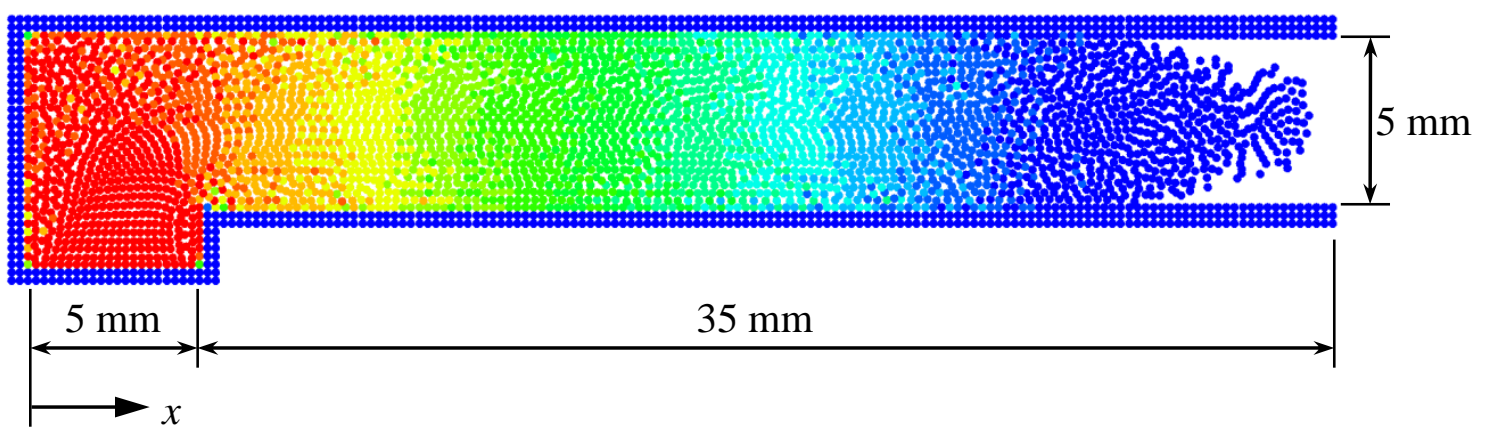

(a) Contour plot

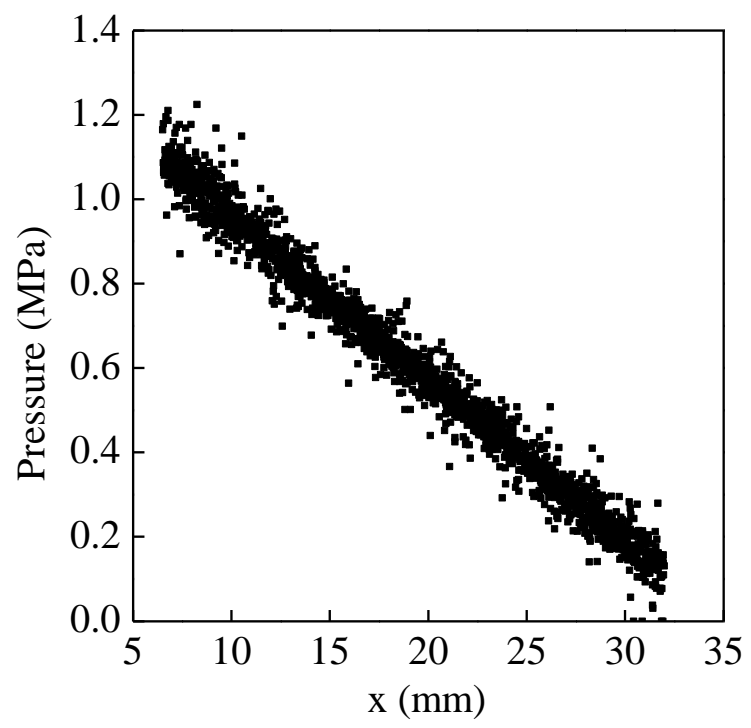

(b) Pressure of particles within $6.5<x<32 \mathrm{~mm}$

Fig. 6 Pressure distribution in a straight flow path at $0.08 \mathrm{~s}$. The pressure decreased linearly. 


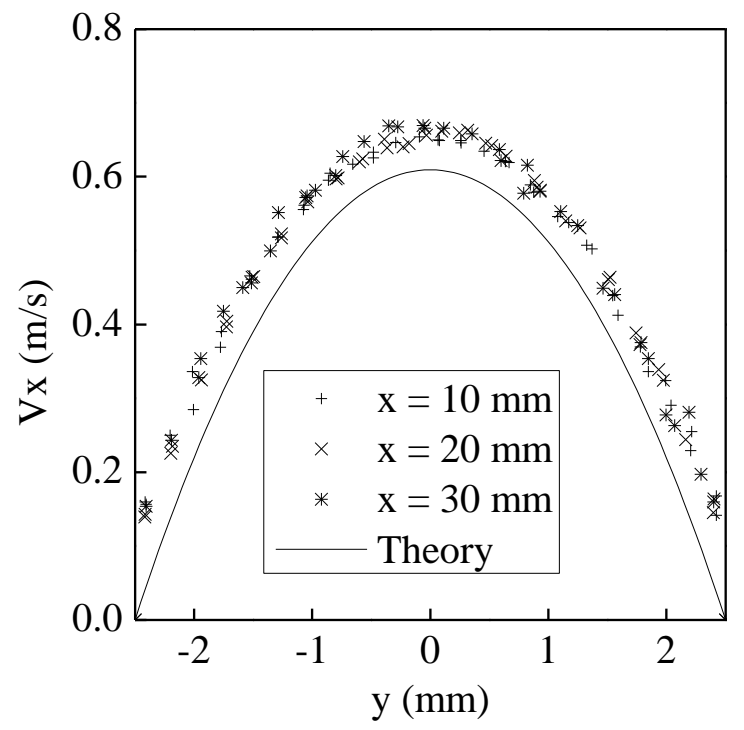

Fig. 7 Comparison of the velocity in the $x$-direction with the prediction and the solution of the Poiseuille flow. 


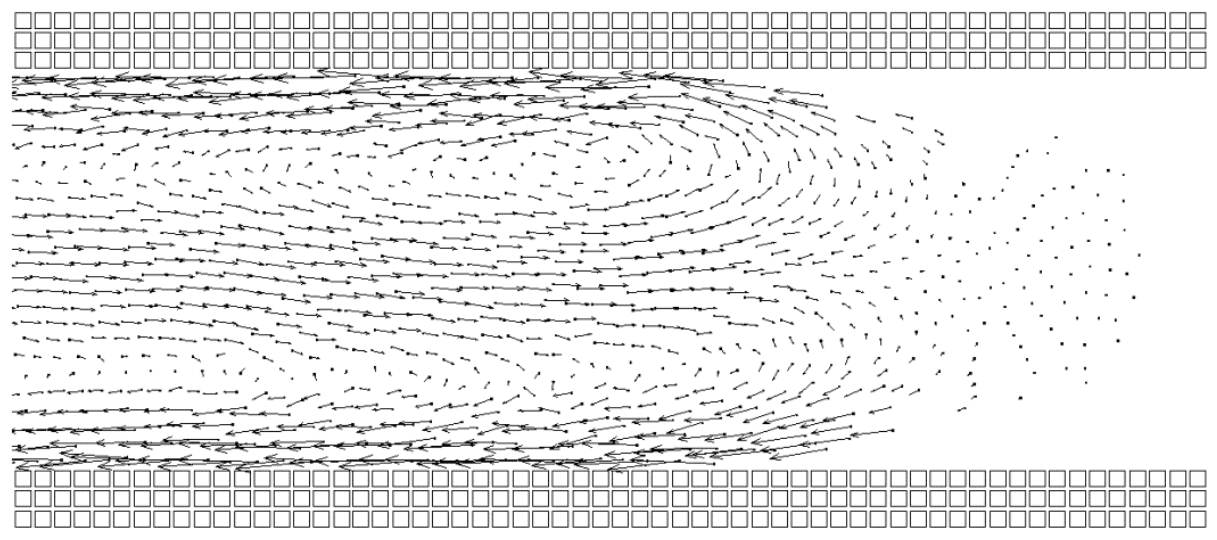

Fig. 8 Distribution of the velocity relative to that of a particle at the flow front, which exhibited fountain flow. 


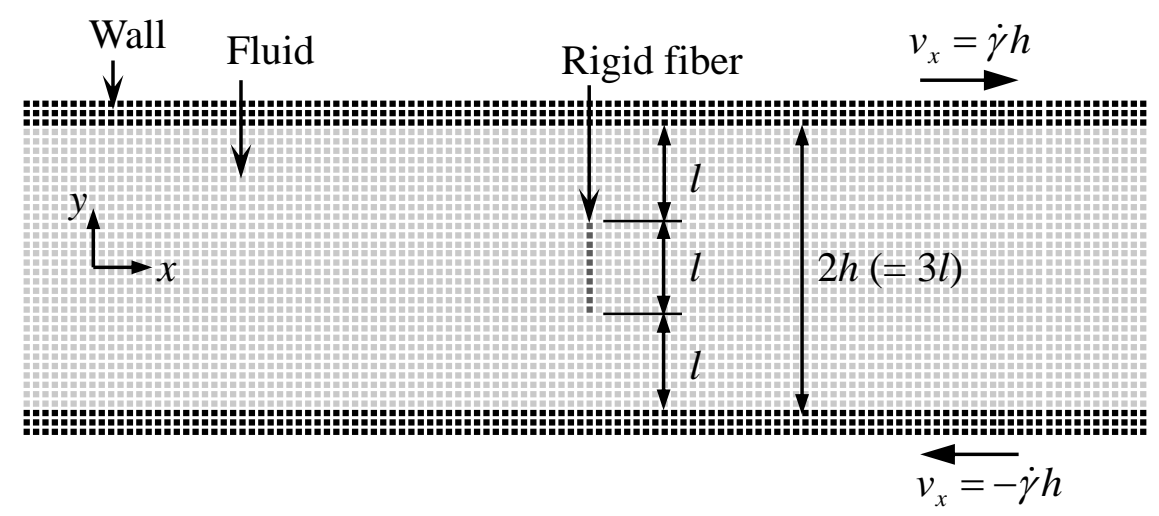

(a) Analytical model

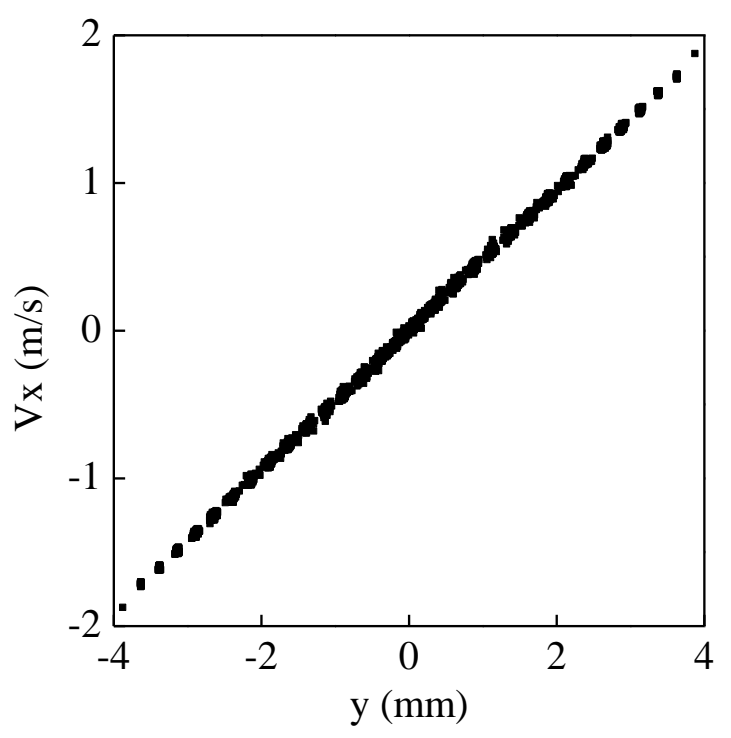

(b) Velocity distribution at $0.02 \mathrm{~s}$

Fig. 9 Analytical model for simple shear flow with a single rigid fiber. The path size was changed by the fiber aspect ratio. 


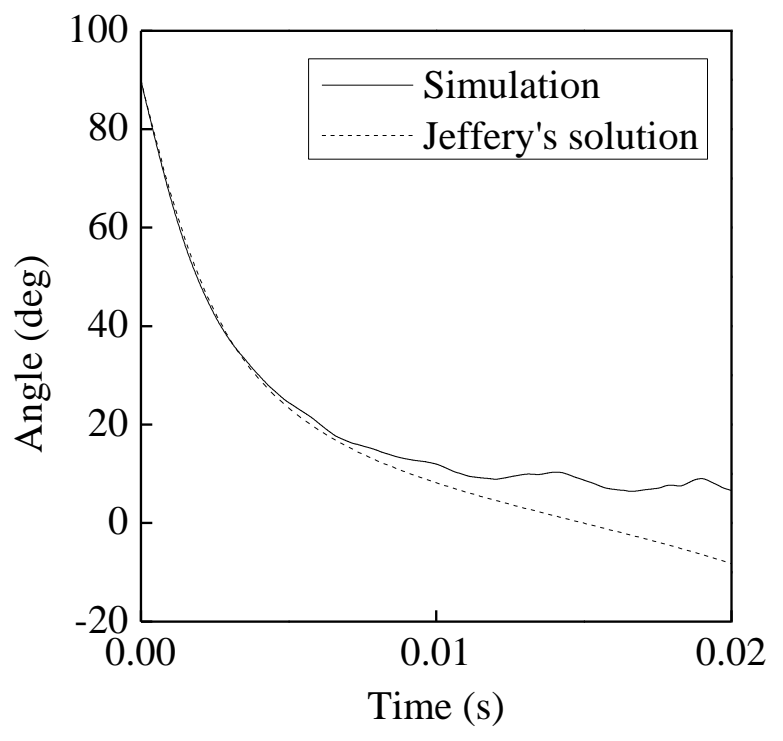

(a) $r_{e}=4$

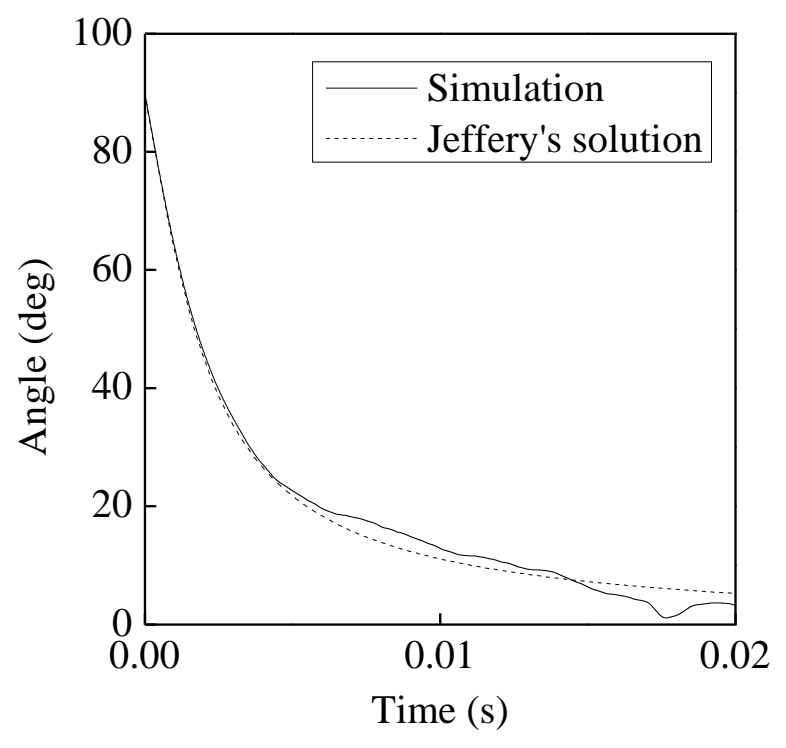

(c) $r_{e}=20$

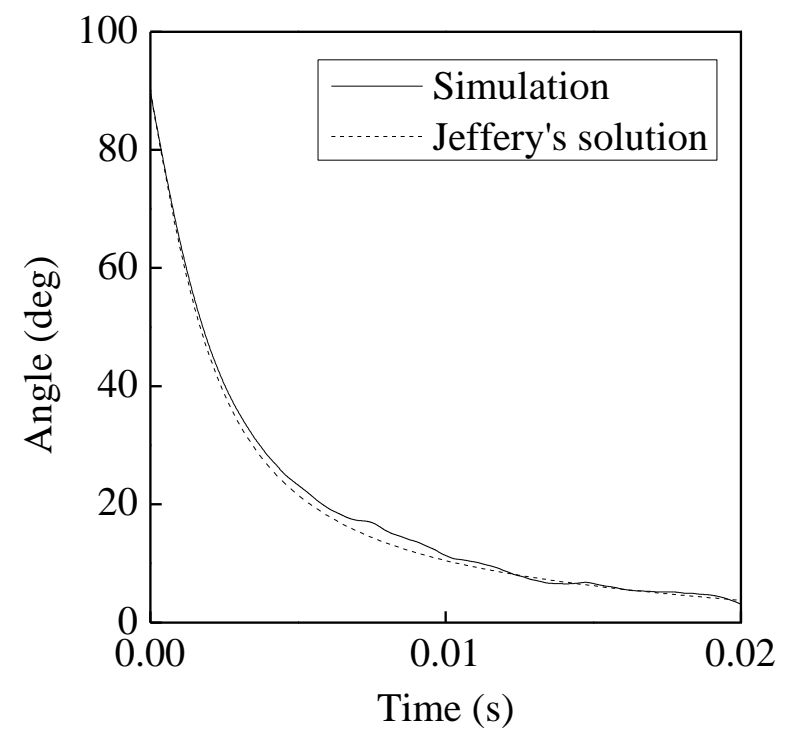

(b) $r_{e}=10$

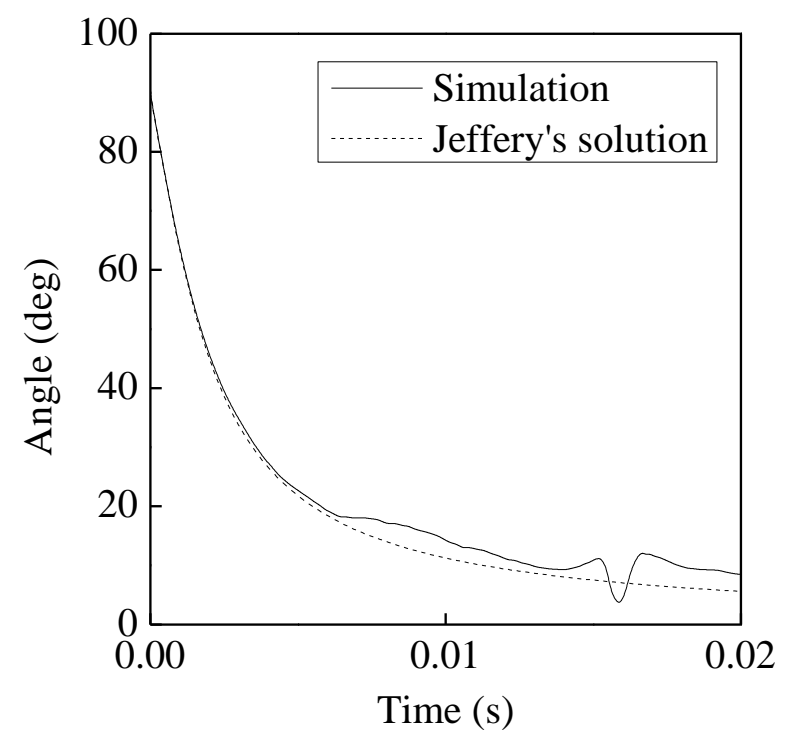

(d) $r_{e}=40$

Fig. 10 Predicted orientation angle in simple shear flow. The Jeffery's solution is also indicated by dashed lines. 


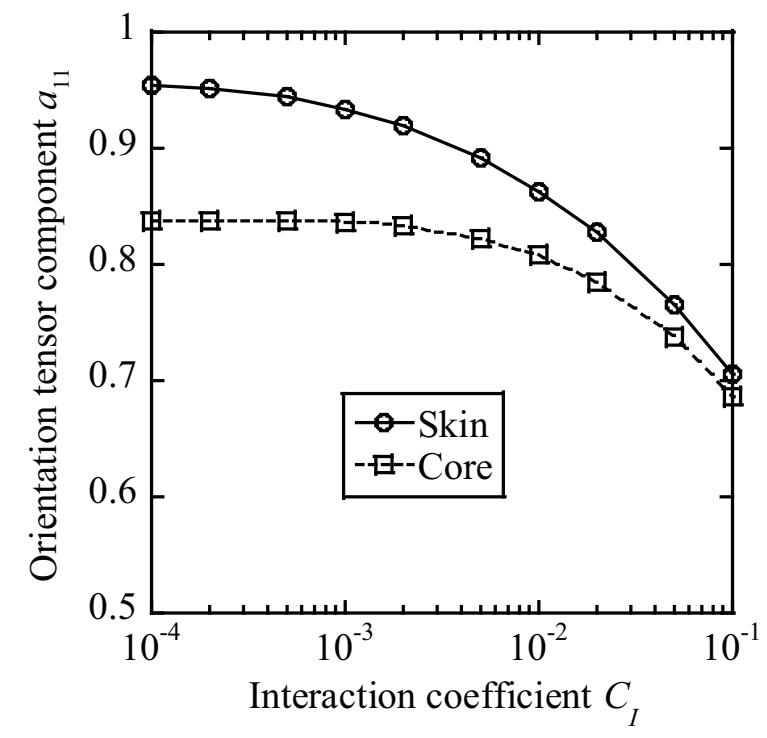

Fig. 11 Change in the orientation tensor component $a_{11}$ against the fiber interaction coefficient calculated at the straight path using the orientation distribution function. 

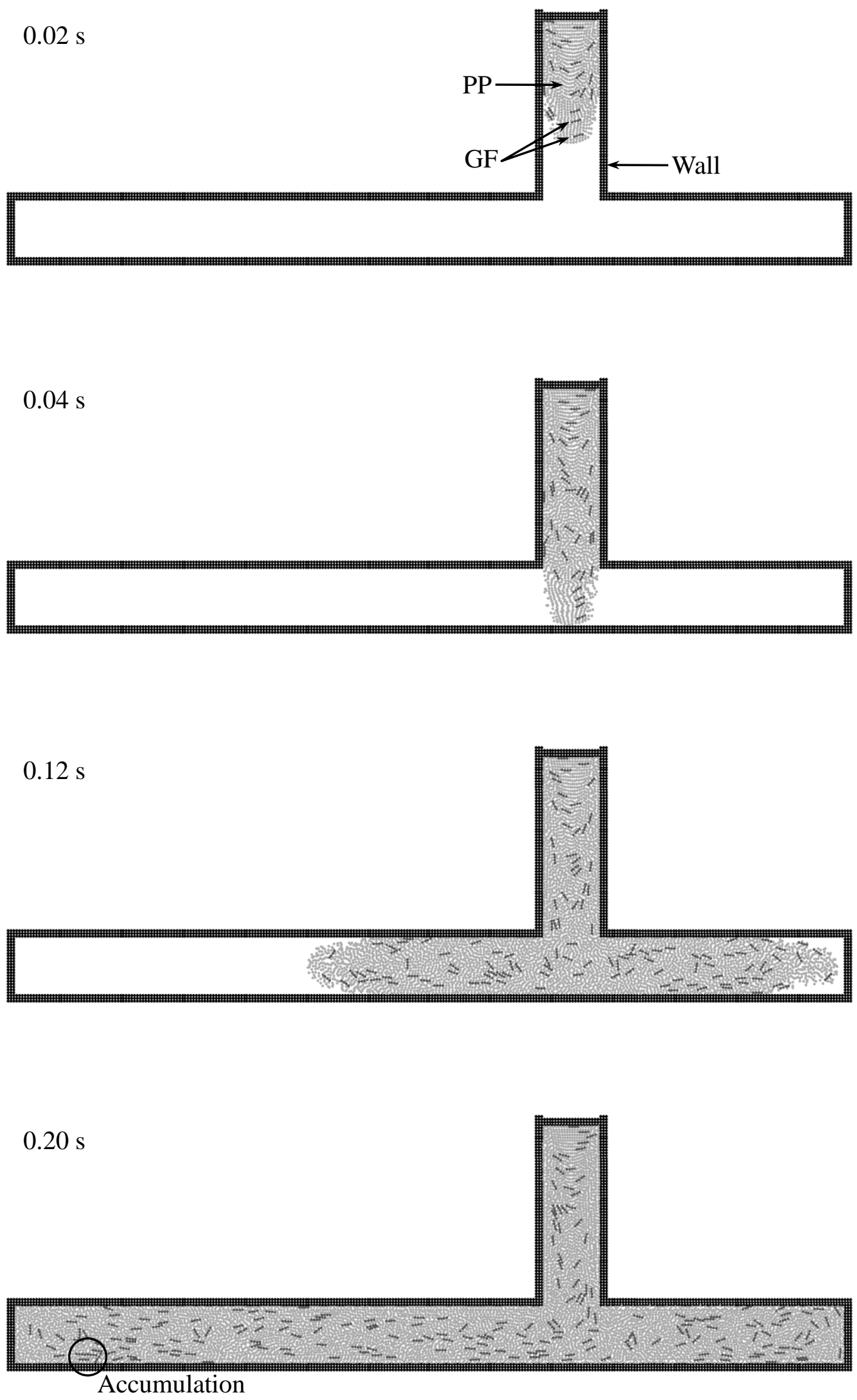

Fig. 13 Snapshots of the mold-filling flow. A fountain flow was generated at the flow-fronts. Fibers accumulated during resin flow. 


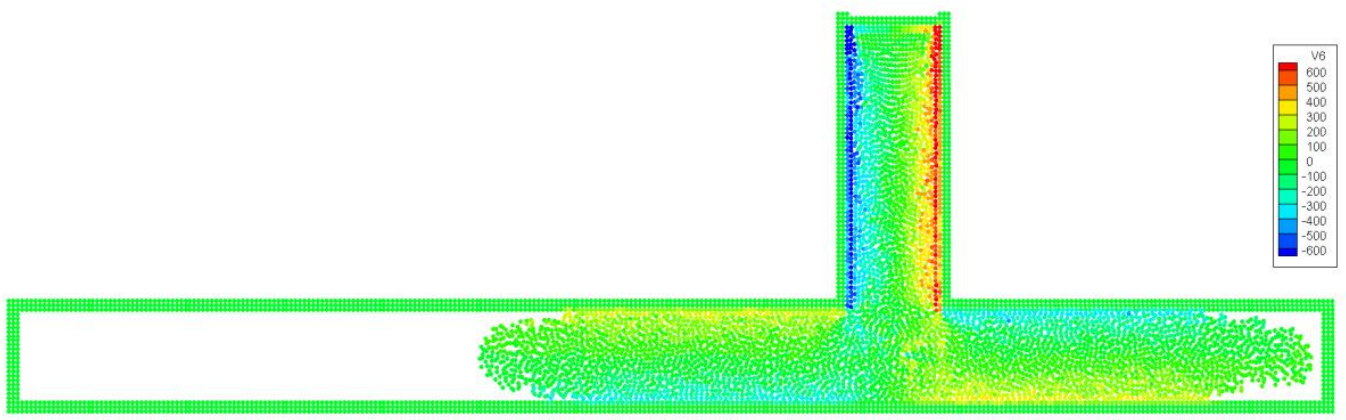

(a) Shear rate

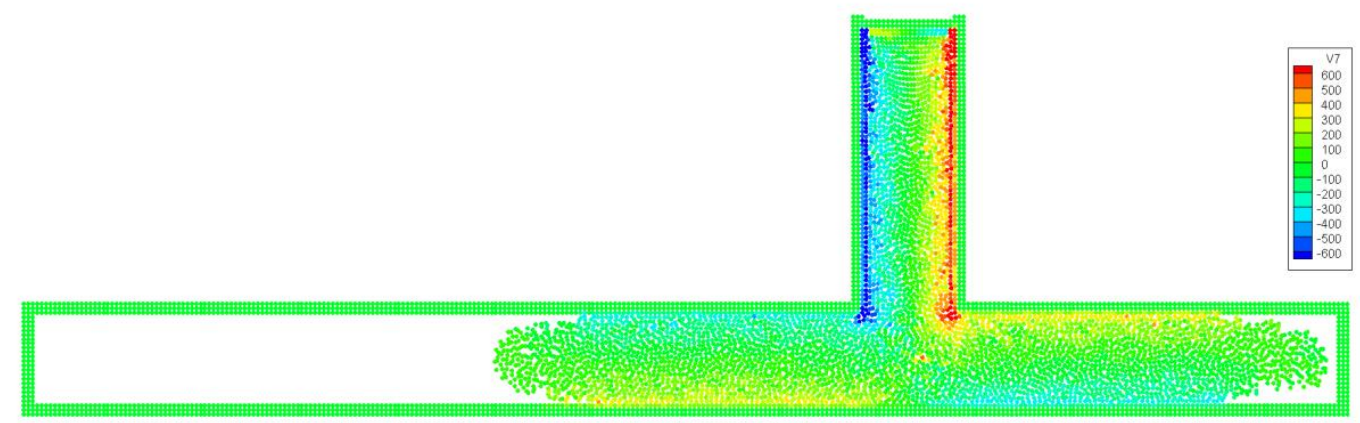

(b) Vorticity

Fig. 14 Distribution of (a) shear rate and (b) vorticity at $0.12 \mathrm{~s}$. 
Figure(s)

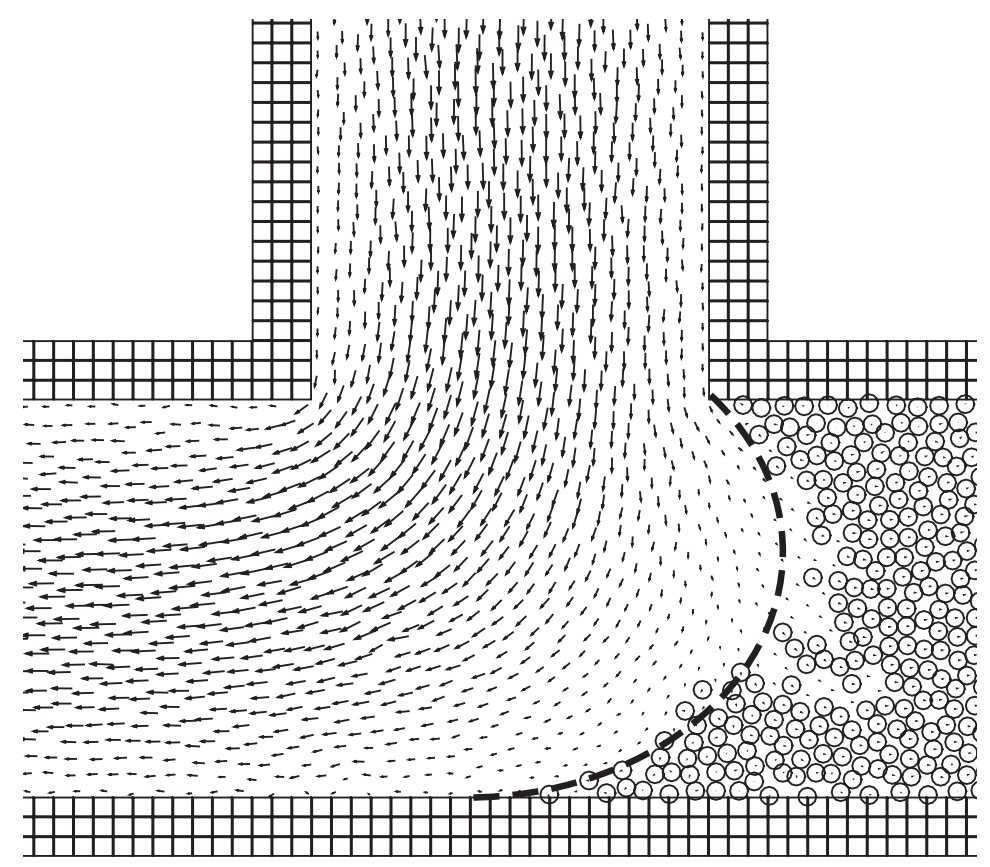

Fig. 15 Velocity vector diagram at $0.16 \mathrm{~s}$ after the $+y$ flow path was filled with resin. Particles with a velocity of $0.025 \mathrm{~m} / \mathrm{s}$ (5\% of the injection speed) are represented by circles. Resin flow almost vanished at the plane indicated by the dashed line. 